\title{
les Hyphomycètes prédateurs de Nématodes : phé- nomène de prédation ; écologie ; utilisation en lutte biologique
}

\author{
Michèle PELOILLE
}

I.N.R.A., Station de Pathologie aviaire et de Parasitologie, Centre de Recherches de Tours, F 37380 Monnaie

RESUME

Hyphomycète Nématophage,

Prédateur,

Ecologie,

Lutte biologique.
Les Hyphomycètes prédateurs présentent plusieurs formes de pièges dont les plus élaborés semblent être les plus aptes à faire régresser une population de nématodes. Il n'y a pas de spécificité absolue quant au choix de proies. De nombreux auteurs ont cherché à mettre en évidence l'existence d'une substance inductrice de la formation des pièges.

Ces organismes sont très répandus à travers le monde et paraissent avoir une large ubiquité. Il reste encore de nombreux biotopes à explorer et nous pensons que bien des espèces sont à découvrir.

L'utilisation de ces champignons comme agents de lutte biologique a suscité de nombreux travaux. Des résultats positifs ont été récemment obtenus en France et sont déjà exploités industriellement dans la lutte contre les anguillules parasites des champignonnières et contre certains Meloidogyne parasites des plantes maraîchères.

L'utilisation de ces Hyphomycètes pour éliminer des nématodes zooparasites n'en est qu'à ses débuts. Les déboires enregistrés jusqu'à présent semblent liés, en partie tout au moins, à des connaissances insuffisantes de la biologie et de l'écologie de ces organismes.

\section{SUMMARY}

Nematode trapping fungi, Hyphomycetes,

Predatory,

Ecology,

Biological control.
Bibliographical review: Nematode-trapping fungi: Predatory phenomenon, ecology, use in biological control

Since more than a century, scientists have been interested by nematode-trapping Hyphomycetes, but the systematic study of these organisms was not thoroughly undertaken before DRECHSLER's works. Nematode-trapping fungi develop different kinds of traps; the most elaborated (constricting rings) seems to be the most effective. Different works concerning the existence of a substance inducing trap formation have been done. Pramer \& Stoll named "nemine" such a substance.

Nematode-trapping fungi are very widespread in the world and seem to be ubiquitous. However many biotopes have not yet been explored and several species are probably to be discovered.

Many works have been induced by the possible use of predatory Hyphomycetes in biological control. In France two positive results have been recently obtained and are already commonly used in the biological control of nematode parasites of cultivated mushrooms and of vegetables.

The use of nematode-trapping fungi against animal parasites is just at its beginning. It seems that discrepancies in the results so far obtained are due, at least for a part, to an insufficient knowledge of the biology and ecology of these organisms.

\section{INTRODUCTION}

Bien que connus depuis un peu plus d'un siècle, les Hyphomycètes prédateurs de nématodes n'ont été étudiés d'une manière systématique que depuis les travaux de DRECHSLER (1937). Les observations qui se rapportent à ces organismes sont dispersées à travers le monde ; il nous a paru intéressant d'en regrouper une partie dans cet article. Hormis le livre attrayant et remarquablement illustré de $G$. BARRON (1977), il n'existe pas, à notre connaissance, de synthèse récente. Nous n'aborderons pas ici l'étude de la systématique des champignons prédateurs ; cette partie très importante fera l'objet d'un autre article. Notons seulement que les Hyphomycètes prédateurs sont des champignons imparfaits qui sont classés en 8 genres regroupant environ 112 espèces.

\section{PHÉNOMÈNE DE PRÉDATION}

Les champignons prédateurs sont habituellement des saprophytes décomposant les produits végétaux, mais ils possèdent la faculté de capturer des nématodes au moyen de pièges plus ou moins élaborés, allant de l'hyphe indifférenciée à des structures plus complexes, tels les anneaux constricteurs. L'induction de la phase saprophyte vers la phase prédatrice est déclenchée par plusieurs facteurs que 
nous étudierons après la description des différents appareils de capture.

\section{Types et formes de pièges}

Les appareils de capture sont de 2 types : pièges passifs et pièges actifs. Les premiers sont les plus répandus et se présentent sous différentes formes.

\section{a) Les hyphes collantes indifférenciées}

sont le type le plus primitif. Elles capturent les nématodes par adhésion à l'aide de la substance collante dont elles sont recouvertes. On les rencontre chez Tridentaria implicans (DRECHSLER, 1937), chez Arthrobotrys anomala (BARRON \& DAVIDSON, 1972) et chez $A$. botryospora (BARRON, 1979) ; chez ces 2 dernières espèces, les hyphes collantes sont généralement associées à des arceaux collants à 3 dimensions (fig. 1).

\section{b) Les arceaux collants tridimensionnels}

sont la forme de piège la plus répandue. Un rameau, issu latéralement d'un filament mycélien, croît puis s'anastomose avec l'hyphe d'origine, formant une boucle. De cette $1^{\text {ère }}$ anse, naissent d'autres branches qui se dirigent dans des plans différents de celui qui renferme la $1^{\text {ère }}$ boucle et s'anastomosent avec celle-ci. Le phénomène se reproduit plusieurs fois et il se constitue ainsi un réseau formé de plusieurs arceaux situés dans les 3 dimensions de l'espace. Ces arceaux sont entièrement tapissés d'une substance collante à laquelle adhèrent les nématodes qui viennent à son contact. Ce type de piège a été décrit par VORONINE en 1864 pour la $1^{\text {ère }}$ fois, sans que la fonction en ait été comprise. C'est ZOPF (1888) qui, le premier, a observé la
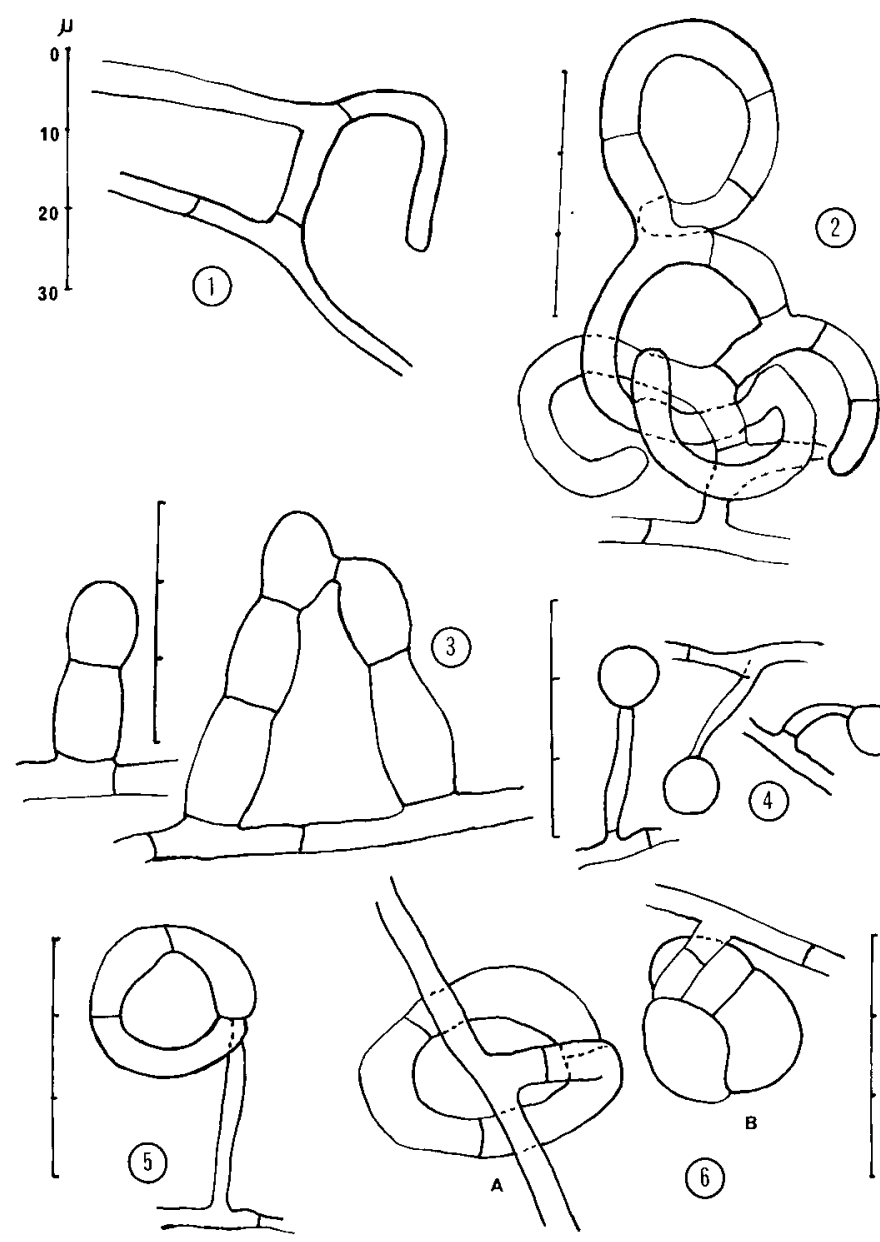

\section{Figure 2 \\ Arceaux collants tridimensionnels. \\ Three-dimensional adhesive net.}

Figure 3

Tubercules collants.

Adhesive branches.

\section{Figure 4}

Boutons collants.

Adhesive knobs.

\section{Figure 5}

Anneaux à trois cellules.

Non constricting ring.

\section{Figure 6}

Anneaux constricteurs.

A : ouvert

$B$ : fermé.

Constricting ring.

A : open

$B$ : closed. 
de la lumière de l'anneau, y enfilent leur extrémité et y demeurent bloqués. On les trouve chez Dactylella asthenopaga (DRECHSLER, 1937) et Dactylella lobata (DUDDINGTON, 1951), comme unique moyen de capture; par contre, chez Dactylaria candida (Nees) (SACCARDO, 1886), Dactylella leptospora (DRECHSLER, 1937) et Monacrosporium ellipsosporum (Preuss) (COOKE \& DICKINSON, 1955), ils sont associés à des boutons collants (fig. 5).

Les pièges actifs sont représentés par des anneaux constricteurs. Il s'agit de 3 cellules arquées et anastomosées, portées par un court pédoncule issu perpendiculairement du mycélium. Les cellules de ce dispositif sont plus larges que celles des anneaux précédemment décrits. La particularité de ce piège réside dans son mode de fonctionnement : les cellules sont sensibles au contact, uniquement à leur face interne (COMMANDON \& DE FONBRUNE, 1939) ; le passage d'un ver à travers l'anneau excite les cellules, entraînant leur gonflement brusque et simultané, en moins d'un dixième de seconde, après une phase de latence d'1 à 2 secondes. La lumière de l'anneau s'obstrue et le nématode se trouve fermement enserré, sans aucune possibilité d'évasion (COUCH, 1937 ; MULLER, 1958 ; RUDEK, 1975 ; DowsETT et al., 1977). L'étude du fonctionnement de ces anneaux a fait l'objet du premier film en micro-cinéma, réalisé en France par COMANDON \& DE FONBRUNE (1939).

On rencontre ces anneaux constricteurs dans les genres Arthrobotrys (A. anchonia DRECHSLER 1954), Dactylaria et Monacrosporium (Dactylaria brochopaga DRECHSLER 1937, M. stenobrochum (Drechsler) SUBRAMANIAN, 1963) (fig. 6).

\section{Mode d'invasion du nématode capturé}

Quel que soit le piège, le mode d'invasion du ver par le champignon est toujours le même. Après un laps de temps plus ou moins long, pendant lequel le nématode piégé se débat violemment, le champignon pénètre à l'intérieur de sa capture en en perforant la cuticule. Il développe ensuite un bulbe d'infection qui a été comparé à l'haustorium de certains champignons parasites et à partir duquel des hyphes trophiques évoluent et envahissent progressivement le ver, en absorbant son contenu, provoquant sa mort en quelques heures. La capture du nématode par Arthrobotrys oligospora a été étudiée et remarquablement illustrée par NORDBRING-HerTZ \& STĂLHammaR-CARLEMaLm, 1978.

\section{Spécificité du piégeage}

L'étude de la bibliographie met en évidence le fait qu'un Hyphomycète prédateur est capable de capturer différents nématodes. Des études réalisées par exemple avec Arthrobotrys oligospora montrent que ce champignon est capable de s'attaquer à des nématodes libres : Rabditis, Mononchus, Cephalobus... (COMMANDON \& DE FonBrune, 1938); à des nématodes parasites du mouton: Haemonchus contortus (VIRAT \& PELOILlE, 1977), Trichostrongylus axei (PANDEY, 1972), à des nématodes parasites des bovins : Ostertagia ostertagi (PANDEY, 1972), ou encore à des nématodes parasites des racines de bégonias (DESCHIENS et al., 1943).

NORDBRING-HERTZ \& MATTIASSON (1979) ont montré qu'une liaison biochimique s'établit entre le piège formé par le champignon et la cuticule du nématode attaqué. Cette liaison se fait entre la lectine et les carbohydrates présents à la surface du nématode. Cependant cette réaction n'est pas spécifique puisqu'elle se réalise de la même façon avec les membranes des globules rouges.

La spécificité du piégeage semble donc assez faible.

L'état actuel de nos connaissances rend nécessaire la mesure de l'efficacité du champignon étudié vis-à-vis du nématode à détruire avant de l'utiliser en tant qu'agent de lutte biologique.

\section{Apparition des pièges}

L'étude des phénomènes qui président au déterminisme de l'apparition des pièges sur un mycélium d'Hyphomycète prédateur n'a commencé qu'en 1937 avec les études réalisées par $\mathrm{COUCH}$. Il est le premier à avoir essayé de stimuler la formation des organes de capture in vitro; il obtint des résultats positifs, en faisant varier le $\mathrm{pH}$ des cultures.

Des essais empiriques réalisés en ajoutant à ces milieux des extraits ou des broyats d'origines diverses, appartenant aussi bien au monde végétal qu'animal, ont été réalisés par ROUBAUd \& DESCHIEnS 1939, DESCHIENS \& LAMY 1942. LAMY, en 1956, montre qu'une substance capable de déclencher la formation des dispositifs de capture existe dans divers extraits d'helminthes et qu'elle est thermolabile et cryostable.

En 1957, LAWTON, observant la formation spontanée de pièges en culture pure, pense que ces champignons élaborent " une substance formatrice d'anneaux" qui pourrait être de nature complexe.

A partir de 1959, PRAMER \& STOLL nomment « némine» un produit métabolique d'origine animale (obtenu à partir de nématodes) et responsable de la formation de pièges. L'activité de cette substance a été étudiée par WINKLER et al. (1961). Ces travaux ont été repris par PRAMER \& KUYAMA (1966) qui pensent que cette "némine " pourrait être un peptide de faible poids moléculaire ou même un acide aminé. WOOTON \& PRAMER (1966) ont isolé et purifié, par dialyse et chromatographie, 3 composants provoquant la formation des pièges chez Arthrobotrys conoides (DRECHSLER, 1937). Ces corps présentent une migration semblable à celle de la valine, de la leucine et de l'isoleucine. Ces recherches ont été complétées par NORDBRING-HERTZ (1973), puis NORDBRING-HERTZ \& BRINCK (1974). Elles ont d'abord montré que la valine a un rôle clé dans l'induction de la formation des pièges et que, dans tous les composés qui déterminent l'apparition de ces pièges, se trouvent la leucine, la valine, l'isoleucine, la proline et la tyrosine. $\mathrm{La}$ position de la valine dans la molécule ne semble pas présenter d'importance pour l'activité.

Cependant, si l'on incrimine une ou plusieurs substances morphogénétiques dans la formation des pièges, il semble bien qu'un certain nombre de facteurs chimiques ou physico-chimiques aient, soit par eux-mêmes, soit par association avec la «némine », la faculté de déclencher l'apparition des appareils de capture. Ainsi, COUCH (1937) réalise le développement des pièges en augmentant l'acidité du milieu. BARTNICKI-GARCIA et al. (1964) observent que la teneur de l'air en $\mathrm{CO}_{2}$ a une influence sur l'intensité de la production des pièges en présence de "némine». NORDBRING-HERTZ (1968) a montré qu'en milieu pauvre, il y a formation spontanée de pièges, alors que celle-ci n'a pas lieu en milieu riche. Les nématodes morts ne déclenchent pas l'apparition des pièges, ce qui permet de penser qu'à côté de la "némine » intervient un autre facteur probablement lié à l'état vivant. Il pourrait s'agir de métabolites azotés excrétés par les nématodes, comme le suggèrent les 
résultats obtenus par SOPRUNOV (1958). D'autre part, la nature de la source de carbone a une influence sur la capacité du champignon à capturer ces nématodes et le nombre de pièges obtenus (HAYES \& BLACKBURN, 1966 ; JAMES \& NOWAKOWSKI, 1968).

Ainsi, le déterminisme de l'apparition des pièges semble lié à plusieurs facteurs de nature biochimique ou physicochimique (conditions générales de nutrition du champignon, substances plus ou moins spécifiques, dont la «némine», stimuli de contact) pouvant intervenir ensemble ou indépendamment les uns des autres, la part revenant à chacun d'entre eux n'étant pas déterminée. Les champignons possédant, comme organe de capture, des arceaux collants, peuvent être considérés comme des saprophytes bons compétiteurs, capables d'utiliser l'amidon et la cellulose, les nitrates, les nitrites et les sels ammoniacaux inorganiques (Satchuthananthavale \& CoOKe, 1967, a-b-c). Ceux qui possèdent des anneaux constricteurs ne peuvent pas utiliser l'ion $\mathrm{NO}_{2}{ }^{-}$. Toutefois, ces études sont fragmentaires et ne portent que sur un nombre réduit d'espèces, ce qui ne permet pas de dégager des conclusions générales applicables à l'ensemble des organismes de ce groupe.

COOKE (1963) observe que, lors de la décomposition de la matière organique, les espèces se succèdent dans un certain ordre : apparaissent d'abord les Hyphomycètes prédateurs à arceaux collants, puis les espèces à rameaux collants et enfin celles possédant des anneaux à 3 cellules ; les champignons possédant ce type d'appareil de capture semblent être de moins bons saprophytes que ceux ayant des pièges plus simples. Il ajoute à ces observations que les espèces apparues tardivement sont les plus efficaces, parmi celles qu'il a étudiées, pour réduire le nombre de nématodes présents dans le sol. Ce résultat est appuyé par les travaux de JANSSON \& NORDBRING-HERTZ (1979) qui ont éprouvé le pouvoir attractif de 14 champignons prédateurs vis-à-vis d'un nématode : Pangrellus redivirus. En général, l'intensité du pouvoir attractif vis-à-vis des nématodes est plus importante chez les espèces plus obligatoirement prédatrices.

\section{II. ÉCOLOGIE}

Les Hyphomycètes prédateurs sont largement répandus à travers le monde, sous toutes les latitudes et à des altitudes variant de 0 à 2000 mètres. Les recherches effectuées aux U.S.A. sont l'œuvre de DrECHSLER $(1937,1950)$ dans le Maryland, TOLMSOFF (1959) en Orégon, FEDER (1962) en Floride, Géorgie et Caroline du Nord. Pour l'Angleterre, citons les travaux de DudDington (1950, 1951 a et b, 1954), MACKENZIE (1960). Des travaux de recherches de grande envergure ont également été faits en U.R.S.S. (SOPRUNOV \& Galiulina, 1951 ; SoPRUNOV, 1958 ; MEKHTIEVA, 1972). En Australie de l'Ouest, nous trouvons les travaux de TAN-HAN-KWANG (1966) et ceux de Mac CUlloch (1977) dans le Queensland. FoWLER (1970) et WoOD (1973) ont recherché les Hyphomycètes prédateurs de NouvelleZélande ; VERONA \& LEPIDI (1970) ceux d'Italie ; ESTEY \& OLTHOF (1965) ceux du Québec. En Inde, DAS-GUPTA et al. (1964) ont étudié la présence de ces organismes au Bengale, SACHCHIDANANDA \& SWARUP $(1966,1967)$ à Delhi. JAROWAJA (1963) a fait un inventaire de ceux de Pologne, SHEPERD (1961) de ceux du Danemark. En France, nous ne connaissons que les travaux de COMANDON \& DE FONBRUNE (1938) ; c'est pour cette raison qu'en 1976 nous avons entrepris l'étude de la mycoflore prédatrice d'une prairie du Limousin (PELOILle, 1979 et 1981).
Ces organismes ont souvent été recherchés là où les auteurs voulaient les utiliser ensuite comme agents de lutte biologique contre les nématodes parasites des racines de plantes (DUDDINGTON, 1954 ; MANKAU \& ClARK, 1959 ; SACHCHIDANANDA \& RAMAKRISHNAN, 1971) dans les terrains maraîchers. Cependant, on peut les trouver dans les endroits les plus divers comme les eaux saumâtres (JoHNSON \& AUTERY, 1961), le bois flotté recouvert par la mer (COOKE \& DICKINSON, 1965). Les excréments en sont particulièrement riches (JUNIPER, 1956, 1957) mais les meilleures sources paraissent être le bois en décomposition et les mousses.

Dans un travail réalisé dans notre laboratoire (VIRAT, 1977 ; PELOILLE, 1979, 1981), nous avons pu mettre en évidence la plus large distribution d'espèces peu fréquentes ou signalées jusqu'alors comme étant liées à des biotopes particuliers : Monacrosporium salinum (Cooke \& Dickinson) isolé à partir de plantes poussant dans l'eau de mer, ou Candelabrella javanica (Rifai \& Cooke) isolé à Java. Nous avons trouvé dans le Limousin, lieu de notre étude, des espèces décrites en France pour la première fois : Arthrobotrys tortor Jarowaja Duddingtonia flagrans (Dudd.) (Cooke), Candelabrella musiformis (Drechsler) (Rifai \& Cooke), en plus des 2 espèces sus-citées. Nous avons noté également l'abondance d'Arthrobotrys oligospora Fres., ce qui est en accord avec les observations faites à travers le globe.

Nous pensons que nos résultats s'expliquent par le fait que peu d'inventaires de la mycoflore prédatrice ont été réalisés jusqu'à ce jour. Malgré l'important travail déjà accompli dans l'étude de la microbiologie du sol, les interactions qui existent entre les différents organismes présents dans ce milieu restent peu connues. On a cependant montré l'existence d'une compétition entre les champignons présents dans le sol pour l'utilisation des sources d'énergie (COOKE, 1962). Les Hyphomycètes prédateurs possèdent la faculté d'utiliser une source alimentaire complémentaire (les proies qu'ils capturent grâce à leurs pièges) et pourraient donc ainsi échapper à la compétition microbienne dans de nombreux milieux.

\section{UTILISATION EN LUTTE BIOLOGIQUE}

L'utilisation des champignons prédateurs comme moyen de lutte biologique a été envisagée contre les nématodes phytoparasites et les nématodes zooparasites. C'est jusqu'à ce jour le premier point qui a été le mieux exploré et également celui pour lequel les résultats sont les plus encourageants. Cette première orientation s'explique par l'impact économique des importants dégâts infligés aux cultures par les nématodes et aussi par la formation de phytopathologistes que possédaient beaucoup de mycologues.

Les premiers essais d'utilisation remontent à 1938-1939 : LINFORD et ses collaborateurs ont essayé de réduire les populations de nématodes responsables de la maladie dite «des racines noueuses» des plants d'ananas, à Hawaï. Ils obtinrent des résultats positifs en ensemençant le sol avec des cultures de champignons (Dactylella ellipsospora Grove) enrichies de matière organique.

D'autres résultats positifs ont été obtenus depuis : en France, des recherches ont été entreprises par DESCHIENS et al. (1943) pour protéger des bégonias cultivés en pots dans une serre, de l'attaque des phytonématodes. Ils ont utilisé un mélange de Dactylella bembicodes Drechsler et d'Arthro. 
botrys oligospora Fresenius. Le nombre de galles est passé de 85 par plant pour les témoins à 5 pour les plants traités.

En U.R.S.S., des travaux analogues ont été entrepris par SOPRUNOV (1952, 1953), SHIPINOVA (1954, 1962), KONDAKOVA (1960), MEKHTIEVA et al. (1967). Les résultats obtenus sont proches de ceux des Français.

Aux Etats-Unis, des études ont été menées dans la perspective d'une lutte contre les parasites de citronniers, en particulier contre Tylenchus semipenetrans Cobb 1913, (DE WOOLF et al., 1954 ; DE WoOLF et al., 1955). MANKAU \& MINTEER (1962) ont prouvé que la population de Tylenchus est réduite à la suite d'apports de matières organiques et qu' Harposporium anguillulae Lohde, champignon endoparasite, joue un rôle important dans le contrôle de la population des nématodes. TARJAN (1961) a essayé de lutter contre un autre parasite du citronnier : Radopholus similis (Cobb) Thorne. Il a été utilisé Arthrobotrys musiformis Drechsler, hyphomycète prédateur choisi pour sa rapidité à produire un grand nombre de chlamydospores in vitro. Il a travaillé en serre et dans les champs, sur de jeunes plants et sur des arbres, avec différents types de matière organique. Il a pu mettre en évidence l'antagonisme existant entre des champignons normalement présents dans le sol (Trichoderma sp. et Gliocladium sp.) et $A$. musiformis. Il conclut cependant en disant que le contrôle biologique des nématodes vivant dans le sol est possible au moyen de champignons prédateurs, bien que ces expériences n'aient pas eu tout le succès escompté.

Des travaux plus récents effectués en France, à la Station de Recherches sur les Nématodes (I.N.R.A. Antibes), ont permis de mettre au point 2 méthodes de lutte biologique contre les anguillules contaminant les champignonnières (CAYROL et al., 1978) d'une part, et d'autre part, contre les Meloidogyne responsables de la formation de galles sur les racines de nombreuses espèces cultivées (CAYROL \& FRANKOWSKI, 1979). Ces 2 méthodes sont maintenant exploitées à l'échelon industriel sous forme d'un mycélium vivant incorporé au sol dans un substrat à base de grains de seigle.

Si d'autres essais eurent des résultats décevants, ceux de HAMS \& WILKIN (1961) par exemple tentant de lutter contre des parasites de racines de pomme de terre, de tomates, de pois et de céréales, on peut cependant admettre que, dans le domaine de la lutte contre les parasites des végétaux, les résultats sont actuellement encourageants.

Les essais d'utilisation des Hyphomycètes prédateurs contre les nématodes parasites d'animaux ont été réalisés essentiellement en France et en U.R.S.S. Les auteurs français (DESCAZEAUX, 1939 a et b ; DESCAZEAUX \& Capelle, 1939 ; Deschiens, 1939 a et b ; Roubaud \& DESCAZEAUX, 1939 ; RoubaUd \& DESCHIENS, 1941) ont mis en évidence in vitro le pouvoir prédateur de certains hyphomycètes vis-à-vis de nématodes parasites de chevaux, ânes et moutons. Ils obtinrent également des résultats positifs sur le terrain.
Les essais in vitro ont d'abord été repris par KORNIENKO et al. (1958) puis, en 1965, par PRIADKo \& Drobichenko. Nous-mêmes, au laboratoire, avons étudié le pouvoir prédateur d'A. oligospora vis-à-vis d'un parasite du mouton : Haemonchus contortus (VIRAT \& PELOILLE, 1977).

Les essais réalisés par les équipes soviétiques sur le terrain ont donné des résultats aléatoires. L'activité des champignons dans la nature, après épandage à la surface du sol, paraît beaucoup moins constante, en raison sans doute de l'effet des conditions climatiques et des problèmes d'interactions entre micro-organismes. Quelques résultats positifs ont cependant été enregistrés (CHAGALINE, 1958, 1959, 1960 a-b-c ; SOPRUNOV, 1958).

Il semble que les résultats variables obtenus jusqu'à présent dans la lutte contre les nématodes zooparasites soient dus, en partie tout au moins, à la méconnaissance de l'écologie et de la physiologie de ces organismes. La plupart des expériences n'ont d'ailleurs porté que sur des nombres très réduits d'animaux et les auteurs eux-mêmes émettent des réserves quant à la généralisation de leurs résultats.

\section{CONCLUSION}

Les différents appareils de capture des Hyphomycètes prédateurs de nématodes et leur mode de fonctionnement sont bien connus. Par contre, les phénomènes qui président à l'apparition de ces pièges le sont moins bien.

A travers la somme de travail déjà effectuée sur ce sujet, il apparaît que les champignons prédateurs de nématodes sont largement répandus à travers le monde. Des espèces, qui semblaient jusqu'à ce jour liées à un biotope bien déterminé, ont été retrouvées dans des biotopes très différents ( $M$. salinum, $C$. javanica). Il semble que leur plus large ubiquité n'ait pas été démontrée auparavant parce que la recherche systématique de leur présence n'a concèné qu'un nombre limité de sites.

L'utilisation en lutte biologique de ces organismes pour faire régresser les populations de Nématodes du sol a attiré l'attention de bien des chercheurs depuis les essais réalisés en 1938 et 1939 par LiNFORD. En France, 2 espèces du genre Arthrobotrys sont utilisées à l'échelle industrielle pour réduire les populations d'anguillules en champignonnières et en cultures maraîchères.

De nombreux essais ont été tentés pour lutter contre les nématodes parasites des animaux : les résultats sont moins concluants et il n'y a pas de travaux plus récents que ceux de Drobischenko PRIADKo (1969). Une meilleure connaissance de l'écologie et de la biologie de ces organismes semble indispensable si l'on veut réaliser des expérimentations dans des conditions plus favorables.

Reçu le 12 novembre 1980. Accepté le 21 janvier 1981.

\section{RÉFÉRENCES BIBLIOGRAPHIQUES}

Barron G. L., 1977. The nematode destroying fungi. Topics in Mycology no 1. Can. Biol. Publ. Guelph, 140 p.

Barron G. L., 1979. Nematophagous fungi : a new Arthrobotrys with non septate conidia. Can. J. Bot., 57, 1371-1373.

Barron G. L., Davidson J. G. N., 1972. Nematophagous hyphomycetes: Arthrobotrys anomala sp. nov. Can. J. Bot., 50, 1173-1174.

Bartnicki-Garcia S., Eren J., Pramer D., 1964. Carbon dioxyde dependent morphogenesis in Arthrobotrys conoides. Nature, 204, 804.

Cayrol J. C., Frankowski J. P., 1979. Une méthode de lutte biologique contre les Nématodes à galles des racines appartenant au genre Meloidogyne. «P.H.M. Rev. hort. », 193, 15-23.
Cayrol J. C., Frankowskí J. P., Laniece A., D'Hardemare G., Talon J. P., 1978. Contre les Nématodes en champignonnière : mise au point d'une méthode de lutte biologique à l'aide d'un hyphomycète prédateur : Arthrobotrys robusta, souche « Antipolis » (Royal 300). "P.H.M. Rev. hort.", 184, 23-30.

Chagaline S. F., 1958. (Influence d'une administration unique par voie orale des conidies du champignon prédateur Arthrobotrys oligospora Fres. sur la quantité de larves de nématodes dans les fèces de mouton) (en russe). Izv. Akad. Nauk Turkmen SSR, 6, 105-107.

Chagaline S. F., 1959. (Méthode de lutte biologique contre les helminthoses en élevage) (en russe). C.X. Turkmen, 2, 38-41. 
Chagaline S. F., 1960a. (Étude comparative de l'efficacité de deux champignons prédateurs : Arthrobotrys oligospora Fres. et Arthrobotrys arthrobotryoides $v$. indolens sur les larves de nématodes pathogènes du cheval) (en russe). Trud. Inst. Zool. Paras. Akad. Nauk Turkmen S.S.R., 5, 225-230.

Chagaline S. F., 1960b. (Destruction des larves du sous-ordre des strongylata par les champignons prédateurs Arthrobotrys oligospora Fres. et Arthrobotrys arthrobotryoides v. indolens dans les fèces du cheval) (en russe). Trud. Inst. Zool. Paras, Akad. Nauk Turkmen S.S.R., 5, 232-236.

Chagaline S. F., 1960c. (Champignons prédateurs pour la prophylaxie des Strongyloses du cheval et du mouton en Turkmenistan) (en russe). Thèse candidat vétérinaire Askhabad.

Cobb N. A., 1913. Notes on Mononchus and Tylenchulus. J. of the Wash. Acad. of Sci., 3, 287-288.

Comandon J., Fonbrune P. (de), 1938. Recherches expérimentales sur les champignons prédateurs de nématodes du sol. Conditions de formation des organes de capture. C.R. hebd. Séances Acad. Sci. Paris, 129, 619-625.

Comandon J., Fonbrune P. (de), 1939. De la formation et du fonctionnement des pièges de champignons prédateurs de nématodes. Recherches effectuées à l'aide de la micromanipulation et de la cinématographie. C.R. hebd. Séances Acad. Sci. Paris, 208, 304-305

Cooke R. C., 1962. The ecology of nematode-trapping fungi in the soil. Ann. appl. Biol., 50, 507-513.

Cooke R. C., 1963. Succession of Nematophagous fungi during the decomposition of organic matter in the soil. Nature, 197, 205.

Cooke R. C., 1969. Two nematodes trapping hyphomycetes Duddingtonia flagrans gen. com. nov. and Monacrosporium mutabilis sp. nov. Trans. Br. mycol. Soc., 53, 315-319.

Cooke R. C., Dickinson C. H., 1965. Nematodes trapping species of Dactylella Monacrosporium. Trans. Br. mycol. Soc., 48, 621-629.

Corda A. C. J., 1839. Pracht Flora, europaischer Schimmelbildungen Leipzig u. Dresder. p. 43

Couch J. N., 1937. The formation and operation of the traps in the nematode catching fungi Dactylella bembicodes Drechs. J. Elisha Mitchell Sci. Soc., 53, 301-309.

Das-Gupta S. N., Shome U., Shome K., 1964. Preliminary report on predacious fungi in India. Curr. Sci., 33, 380-381.

Descazeaux J., 1939a. Stérilisation biologique de crottins parasités par des larves de Nématodes. Bull. Acad. vét. Fr., 12, 136-139. Descazeaux J., 1939b. Action des champignons Hyphomycètes prédateurs sur les larves de certains nématodes parasites de ruminants. Bull. Soc. Path. exot., 32, 457-459.

Descazeaux J., Capelle, 1939. Contribution à l'étude des champignons prédateurs de larves de Nématodes parasites des animaux domestiques. Bull. Acad. vét. Fr., 12, 284-288.

Deschiens R., 1939a. Capture et destruction de larves de strongylidés du singe et du bœuf par des Hyphomycètes. Bull. Soc. Path. exot., 32, 394-398.

Deschiens R., 1939b. Considérations relatives à la destruction des larves de Nématodes parasites par des Hyphomycètes prédateurs. Bull. Soc. Path. exot., 32, 459-464.

Deschiens R., Lamy L., 1942. Sur les facteurs déterminant l'apparition des pièges chez les Hyphomycètes prédateurs de Nématodes. C.R. hebd. Séances Acad. Sci. Paris, 215, 450-452.

Deschiens R., Lamy L., Vautrin E., 1943. Essai de pratiques de prophylaxie de l'anguillulose des végétaux par l'emploi d'Hyphomycètes prédateurs. C.R. hebd. Séances Acad. Sci. Paris, 216, 539-541.

Dowsett J. A., Reid J., Van Caeseele L., 1977. Transmission and scanning electron microscope observations on the trapping of nematodes by Dactylaria brochopaga. Can. J. Bot., 55 (22), 2945-2970.

Drechsler C., 1937. Some hyphomycetes that prey on free living terricolous Nematodes. Mycologia, 29, 447-552.

Drechsler C., 1950. Several species of Dactylella and Dactylaria that capture free living Nematodes. Mycologia, 42, 1-79.

Drechsler C., 1954. Some Hyphomycetes that capture eelworms in Southern States. Mycologia, 46, 762-782.

Drobischenko N. I., Priadko E. I., 1969. (Champignons helminthophages dans la lutte contre les larves des nématodes parasites des animaux) (en russe). Travaux d'helminthologie au KakhastanAlmanata. Izd. A.N. Kaz. SSR, 129-134.
Duddington C. L., 1950. Further records of british predacious fungi I. Trans. Br. mycol. Soc., 33, 209-214.

Duddington C. L., 1951a. Further records of british predacious fungi II. Trans, Br. mycol. Soc., 34, 194-209.

Duddington C. L., 1951b. The ecology of predacious fungi I. Preliminary survey Trans. Br. mycol. Soc, 34, 322-331.

Duddington C. L., 1954. Nematode destroying fungi in agricultural soils Nematodes. Nature, 173, 500-501.

Estey R. H., Olthof Th. M. A., 1965. The occurrence of nematophagous fungi in Quebec. Phytoprotection, 46, 14-17.

Feder W. A., 1962. Nematophagous fungi recovered around Highlands, North Carolina. Plant Dis. Rep., 46, 872-883.

Fowler M. A., 1970. New Zealand predacious fungi. N.Z. J. Bot., 8, 283-302.

Fresenius G., 1850. Beiträge zur Mykologie, 1, 18-19. Franckfurt a. Main.

Grove W. B., 1884. New or note worthy fungi : Part I. J. Bot., 22, 199.

Hams A. F., Wilkin G. D., 1961. Observations on the use of predacious fungi for the control of Heterodera spp. Ann. Appl. Biol., 49, 515-523.

Hayes W. A., Blackburn F., 1966. Studies on the nutrition of Arthrobotrys oligospora and Arthrobotrys robusta. II. The predacious phase. Ann. appl. Biol., 58, 51-60.

James A. W., Nowakowski R. J., 1968. The effect of carbon source on trap formation by Arthrobotrys conoides. Can. J. Microbiol., 14, 1260-1261.

Jansson H. B., Nordbring-Hertz B., 1979. Attraction of Nematodes to living mycelium of nematophagous fungi. J. gen. Microbiol., 112, $89-93$.

Jarowaja N., 1963. (Preliminary investigations on fungi natura ennemies of eelworms) (en polonais). Biul. Inst. Ochr. Rosl., 21, 189-196.

Johnson T.W., Autery Ch. L., 1961. An Arthrobotrys from brackish water. Mycologia, 53, 432-433.

Juniper A. J., 1956. A study of predacious fungi with particular reference to those occurring in dung. Ph. D. Thesis. University of London 1956.

Juniper A. J., 1957. Dung as a source of predacious fungi. Trans Brit. mycol. Soc., 40, 346-348.

Kondakova E. I., 1960. (Au sujet de la répartition spécifique des champignons prédateurs près de Moscou et étude de la possibilité de leur utilisation dans la lutte biologique contre le nématode gallique en terrain clos) (en russe). Avtoref. Diss. Moscou Univ. Lomonossov, 3-14.

Kornienko Z. P., Tendetnik Y. Y., Tchareiev O. Tch. In Soprunov F. F., 1958. (Predacious hyphomycetes and their application in the control of pathogenic Nematodes). Trad. du russe par S. Nemchonok Israël program for Scientific Translation, Jerusalem 1966. Ashkabad : Izdatel' stvo Akademii Nauk Turkmenskoi SSR, 292 p., 227-232.

Lamy L., 1956. Identité possible des substances actives sur la formation des dispositifs capteurs chez les Hyphomycètes prédateurs et des substances antigéniques des extraits d'helminthes parasites. C.R. hebd. Séances Acad. Sci. Paris, 242, 692-694.

Lawton J. R., 1957. The formation and closure of constricting rings in Nematode catching Hyphomycetes grown in pure culture. J. exp Bot., 8, 50-54.

Linford M. B., Oliveira J. M., 1938. Root injury restricted by a Nematode trapping fungus. Phytopathology, 28, 14-15.

Linford M. B., Yap F., 1939. Rootknot nematode injury restricted by a fungus. Phytopathology, 29, 595-609.

Linford M. B., Yap F., Oliveira J. M., 1939. Reduction of soil populations of the root knot nematode during decomposition of organic matter. Soil Sci., 45, 127-140,

Mac Culloch J., 1977. A survey of nematophagous fungi in Queensland. Queensl. J. agric. anim. Sci., 34, 25-34.

Mackenzie D. W. R., 1960. Predacious fungi in Edinburgh district. Proc. bot. Soc. Edinb., 39, 35-45

Mankau R., Clarck O.F., 1959. Nematode trapping fungi in Southern California citrus soil. Pl. Dis. Rep., 43, 968-969.

Mankau R., Minteer R. J., 1962. Reduction of soil populations of the citrus nematode by the addition of organic materials. PI. Dis. Rep., 46, 375-378. 
Mekhtieva N. A., 1972. (Main results and outlooks of studying predacious fungi in Azerbaïdjan) (en russe). Mikol. Fitopat., 6, 477-482.

Mekhtieva N. A., Zeillanova Ch., Ganiyeva T. A., 1967. (Sur l'utilisation des champignons prédateurs contre les nématodes des galles et étude de leur activité dans le sol) (en russe). Sbor. Nemat. Bolez. Sel'sk. Rast, 101-105.

Muller H. G., 1958. The constricting ring mechanism of two predacious hyphomycetes. Trans. Br. mycol. Soc., 41, 341-364.

Nordbring-Hertz B., 1968. The influence of medium composition and additions of animal origin on the formation of capture organs in Arthrobotrys oligospora. Physiol. Plant., 21, 52-65.

Nordbring-Hertz B., 1973. Peptide induced morphogenesis in the nematode trapping fungus Arthrobotrys oligospora. Physiologia Plant. 29, 223-233.

Nordbring-Hertz B., Brinck C., 1974. Qualitative characterization of some peptides inducing morphogenesis in the nematode trapping fungus Arthrobotrys oligospora. Physiol. Plant., 31, 59-63.

Nordbring-Hertz B., Mattiasson, 1979. Action of a nematode, trapping fungus shows lectin-mediated host-microorganism interaction. Nature, 281, 477-479.

Nordbring-Hertz B., Stolhammar-Carlemalm M., 1978. Capture of nematode by Arthrobotrys oligospora, an electron microscope study. Can. J. Bot., 56, 1297-1307.

Oudemans C. A. J. A., 1885. Aarwinsten voor de flora mycologica van Nederland. 9-10. Ned. Krnidk. Arch. ser., 2, 203-278.

Pandey V.S., 1972. Contribution to the study of gastrointestinal Nematodes of cattle. Doct. Sci. Thesis. University of Liege (Belgium), $192 \mathrm{p}$.

Peloille M., 1981. Etude des Hyphomycètes prédateurs de Nématodes rencontrés sur une prairie du Limousin: Morphologie Physiologie - Fréquence et distribution. Thèse de l'Université de Rennes, $106 \mathrm{p}$.

Peloille M., 1979. Étude des Hyphomycètes prédateurs de Nématod'une prairie du Limousin. Entomophaga 26 (1), 91-98.

Pramer D., Kuyama S., 1963. Symposium on biochemical bases of morphogenesis in fungi II. Nemin and the nematode trapping fungi. Bact. Rev., 27, 282-292.

Pramer D., Stoll N. R., 1959. Nemin : a morphogenic substance causing trap formation by predaceous fungi. Science, 129, 966-967. Priadko E. I., Drobichenko N. I., 1965. (Essai de mise en évidence de l'activité d'une préparation du champignon prédateur Arthrobotrys oligospora envers les larves de Strongles intestinaux du mouton et de nématodes libres du sous-ordre des Rhabditata) (en russe). Mater. Nauchn. Konf., 1, 181-183.

Rifai M. A., Cooke R. C., 1966. Studies on some didymosporous genera of nematode trapping hyphomycetes. Trans. Br. mycol. Soc., 49, 147-168.

Roubaud E., Descazeaux J., 1939. Action de certains champignons prédateurs sur les larves de Strongylidés du cheval. Bull. Soc. Path. exot., 32, 290-294.

Roubaud E., Deschiens R., 1939. Sur les agents de formation des dispositifs de capture chez les Hyphomycètes prédateurs de Nématodes. C.R. hebd. Séances Acad. Sci. Paris, 209, 77-79.

Roubaud E., Deschiens R., 1941. Action des Hyphomycètes prédateurs sur les larves de synthétocaules et de bunostomes. Bull. Soc. Path. exot., 34, 127-130.

Rudek W. T., 1975. The constriction of the trapping rings in Dactylaria brochopaga. Mycopathologie, 55 (3), 193-197.

Saccardo P.A., 1880. Dactylaria purpurella. Michelia, 2, 20. Saccardo P. A., 1886. Sylloge fungorum omnium hucusque cognitorum, 4, 194-195, publiés par l'auteur, Pavie.

Sachchidananda J., Ramakrishnan K., 1971. Nematophagous fungi of agricultural soils. Mycopath. Mycol. appl., 43, 235-245.

Sachchidananda J., Swarup G., 1966. Nematophagous fungi in Delhi soils. India Phytopathol. 19, 279-285.

Sachchidananda J., Swarup G., 1967. Additional nematophagous fungi from Delhi soils. Curr. Sci., 24, 677-678.

Satchuthananthavale V., Cooke R. C., 1967a. Carbohydrate nutrition of some nematode trapping fungi. Nature, 214, 321-322.
Satchuthananthavale V., Cooke R. C., 1967b. Vitamin requirements of some nematode trapping fungi. Trans. Br. mycol. Soc., 50, 221-228.

Satchuthananthavale V., Cooke R. C., 1967c. Nitrogen nutrition of some nematode trapping fungi. Trans. Br. mycol. Soc., 50, 423-428. Sheperd A. M., 1961. Nematode trapping fungi in Danish agricultural soils. Horticultura, 15, 94-96.

Shipinova S. I., 1954. In Soprunov F. F. 1958. Khishchnye gribygifomitzety i ikh primenenie $v$ bor'be $s$ patogennymi nematodami/Predacious Hyphomycetes and their application in the control of pathogenic Nematodes. Traduit du russe par S. Nemchonok Israël program for Scientific Translation, Jerusalem 1966, Ashkhabad: Izdatel'stvo Akademii Nauk Turkmenskoi SSR, 292 p., 241-248.

Shipinova S. I., 1962. Studies on the use of predatory fungi for control of the root knot nematode (en russe). Proc. Sth all. Union Conf. Phyto. helminth., 348-352.

Soprunov F. F., 1952. In Soprunov F. F. 1958. Khishchnye gribygifomitzety $\mathrm{i}$ ikh primenenie $\mathrm{v}$ bor'be $\mathrm{s}$ patogennymi nematodami/Predacious Hyphomycetes and their application in the control of pathogenic Nematodes. Traduit du russe par S. Nemchonok Israël program for Scientific Translation, Jerusalem 1966. Ashkhabad : Izdatel'stvo Akademii Nauk Turkmenskoi SSR, 292 p. 223-252.

Soprunov F. F., 1953. (Application of predacious fungi in the protection of plants against phytonematodes) (en russe). Izv. Akad. Nauk TSSR, 1, 81.

Soprunov F. F., 1958. Khishchnye griby-gifomitzety i ikh primenenie $\mathrm{v}$ bor'be s patogennymi nematodami/Predacious Hyphomycetes and their application in the control of pathogenic Nematodes. Traduit du russe par S. Nemchonok Israël program for Scientific Translation, Jerusalem 1966. Ashkhabad: Izdatel'stvo Akademii Nauk Turkmenskoi SSR, $292 \mathrm{p}$.

Soprunov F. F., Galiulina Z. A., 1951. (Hyphomycètes prédateurs des sols du Turkmenistan) (en russe). Mikobiologiya, 20, 489-499. Subramanian C. V., 1963. Dactylella, Monacrosporium and Dactylina. J. Indian bot. Soc., 42, 291-300.

Tan-Han-Kwang B. S., 1966. Nematophagous fungi from Western Australia. M. Sc. Thesis University of Western Australia, 249 p.

Tarjan A. C., 1961. Attempts at controlling citrus burrowing Nematodes using Nematode trapping fungi. Proc. Soil Crop. Sci., Florida, 21, 17-35.

Tolmsoff W. J., 1959. The isolation of nematode trapping fungi from Oregon soils. Phytopathology, 49, 113-114.

Verona O., Lepidi A. A., 1970. Primi reperti sulla presenza di micromiceti predatori di nematodi in alcuni terreni italiani. Agric. ital., 70, 217-225.

Virat M., 1977. Sur deux hyphomycètes prédateurs de nématodes isolés de prairie et notes sur les genres Candelabrella et Duddingtonia. Rev. de Mycol., 41, 415-426.

Virat M., Peloille M., 1977. Pouvoir prédateur in vitro d'une souche d'Arthrobotrys oligospora Fres. vis-à-vis d'un nématode zooparasite. Ann. Rech. vet., 8, 51-58.

Voronine M. S., 1864. In De Bary : Beiträge zur Morphologie und Physiologie der Pilze. Frankfort sur le Main. p. 29.

Winkler E. J., Kuyama S., Pramer D., 1961. A nemin assay procedure. Nature, 191, 155-156.

Wood F. H., 1973. Nematode trapping fungi from a tussock grassland in New-Zealand. N.Z.J. Bot., 11, 231-240.

Woolf T. A. (de), Klotz L. J., Baines R. C., Moore P. W., 1954. Nematode capturing fungi. Calif. Citrograph., 39, 104.

Woolf T. A. (de), Klotz L. J., Hashimoto S., 1955. Nematophagous fungi in orchards. Phytopathology, 43, 693.

Wooton L. M. O., Pramer D., 1966. Valine induced morphogenesis in Arthrobotrys conoides. Bact. Proc. 66th annual meeting Los Angeles (Ca), $1^{\text {er }}$ au 5 mai, 1966, p. 75. Abstr. no P31 (Amer. Soc. Microbiol.).

Zopf W., 1888. Zur Kenntniss der Infektions-Krankheiten niederer Tiere und Pflanzen. Nova Acta Acad. Caesar. Leop. Carol., 52, 314-376. 